WIS/97/25, EFI-97-31, RI-7-97

hep-th/9707217

\title{
Algebraic Aspects of Matrix Theory on $T^{d}$
}

\author{
S. Elitzur ${ }^{1}$, A. Giveon ${ }^{1}$, D. Kutasov ${ }^{2,3}$, E. Rabinovici ${ }^{1,4}$ \\ ${ }^{1}$ Racah Institute of Physics, The Hebrew University, Jerusalem, 91904, Israel \\ ${ }^{2}$ Department of Physics, University of Chicago, 5640 S. Ellis Ave., Chicago, IL 60637, USA \\ ${ }^{3}$ Department of Physics of Elementary Particles, Weizmann Institute of Science, Rehovot, Israel \\ ${ }^{4}$ Laboratoire de Physique Théorique et Hautes Énergies, URA 280 CNRS, \\ Université Pierre et Marie Curie, Paris, France
}

\begin{abstract}
We study the exceptional $\mathrm{U}$ duality group $E_{d}$ of M-theory compactified on a $d$-torus and its representations using Matrix theory. We exhibit the $E_{d}$ structure and show that $p$ branes wrapped or unwrapped around the longitudinal direction form representations of the U duality group together with other, more mysterious, states.
\end{abstract}




\section{Introduction}

Supersymmetric Yang-Mills theories in various dimensions invariant under sixteen supercharges (SYM) have played an important role in recent developments in field and string theory (see e.g. [1] for a review). Aside from the intrinsic interest in these theories, which is partly due to the fact that the large amount of supersymmetry provides powerful constraints on the dynamics and allows one to study some of the strong coupling phenomena, they are also of interest in string theory for at least two (related) reasons. First, they describe the low energy dynamics on various branes (see e.g. [2] for a review). Second, via the matrix theory conjecture [3, [3] they describe the infinite momentum frame (IMF) dynamics in M-theory.

To study M-theory compactified on a spatial torus $T^{d}$ to $(10-d)+1$ dimensions in the IMF, one needs to consider an $S U(N)$ SYM theory in $d+1$ dimensions on a spatial torus $\tilde{T}^{d}$ which is dual to the original M-theory torus $T^{d}$ [3.5. For $d=1,2$ this prescription has been extensively tested and shown to pass many consistency checks [6-8]. In particular, the U duality group of M-theory on $T^{2}, S L(2, Z)$ [9,10] is manifest. It corresponds to the geometrical symmetry of SYM on the dual torus, $\tilde{T}^{2}$. For $d=3$ there is a new element; part of the $\mathrm{U}$ duality group [9] arises as a quantum $(S L(2, Z))$ Montonen-Olive duality symmetry [11] of $3+1$ dimensional $N=4 \mathrm{SYM}$. One can think of the Montonen-Olive duality of SYM as corresponding to the perturbative T duality of the type IIA string on a two - torus [12, 13, 14].

For $d>3$ SYM is strongly coupled at short distances, and additional data is needed to define the quantum theory. Generically in field theory there are many high energy theories leading to the same low energy dynamics (in this case $d+1$ dimensional SYM) and it is impossible to deduce the former based on knowledge of the latter. However, here due to the large amount of SUSY one may hope that imposing a small number of requirements will suffice to pin down the short distance structure of the theoryl. For $d=4$ [15, 16, 17] and $d=5$ [18,19 there are candidates (at least in principle) for a high energy theory with the right features. Beyond five dimensions the problem remains unsolved; in this note we will describe some properties of this theory for $d \geq 6$. In a theory of extended objects such as M-theory, the number of degrees of freedom necessary for a full description might

1 One natural regulator of $d+1$ SYM is string theory, but that has many additional degrees of freedom (in particular gravity). One is looking for the minimal theory with the right properties. 
increase with compactification. Thus the fully compactified theory is of particular interest, as it may contain the maximal set of degrees of freedom.

To proceed, we need to state the additional requirements we will impose. To be specific, we will consider SYM on a rectangular toru ${ }^{2} \tilde{T}^{d}=\left(S^{1}\right)^{d}$ with the radii of the $d$ circles being $\left(s_{1}, \cdots, s_{d}\right)$; the gauge coupling of the $d+1$ dimensional SYM theory will be denoted by $g$.

Now, if three of the radii $s_{i}$, say $s_{1}, s_{2}$ and $s_{3}$, are much larger than the other $d-3$, then for energies $E<<1 / s_{a}(a=4, \cdots, d)$ the theory looks like $3+1$ dimensional SYM with an effective gauge coupling $g_{\text {eff }}$

$$
\frac{1}{g_{\mathrm{eff}}^{2}}=\frac{W}{g^{2}} ; \quad W=\prod_{a=4}^{d} s_{a}
$$

This theory has an exact Montonen-Olive duality symmetry

$$
g_{\mathrm{eff}} \rightarrow \frac{1}{g_{\mathrm{eff}}} ;\left\{s_{1}, s_{2}, s_{3}\right\} \rightarrow\left\{s_{1}, s_{2}, s_{3}\right\}
$$

It is natural to require the full theory to have the symmetry (1.1) as well. Under this symmetry $g_{\mathrm{eff}} s_{a}(a=4, \cdots, d)$ are invariant, i.e. $s_{a}$ transform as

$$
s_{a} \rightarrow g_{\mathrm{eff}}^{2} s_{a} ; a=4, \cdots, d
$$

Physically, the requirement is that as we turn on the $d-3$ radii $s_{a}$, massive states and interactions come down in energy in precisely the right way to maintain the MontonenOlive duality of $3+1$ dimensional SYM.

Combining (1.1), (1.2), (1.3) we conclude that Montonen-Olive duality on the three torus $T_{i j k}=S_{i}^{1} \times S_{j}^{1} \times S_{k}^{1}$ is the transformation

$$
\begin{aligned}
& g^{2} \rightarrow \frac{g^{2(d-4)}}{W^{d-5}} ; \quad W=\prod_{n \neq i, j, k} s_{n} \\
& s_{\alpha} \rightarrow s_{\alpha} ; \quad \alpha=i, j, k \\
& s_{a} \rightarrow \frac{g^{2}}{W} s_{a} ; \quad a \neq i, j, k
\end{aligned}
$$

2 The restriction to $\tilde{T}^{d}=\left(S^{1}\right)^{d}$ means that we will see only a part of the structure (e.g. only the part of the $\mathrm{U}$ duality group that takes a rectangular torus to a rectangular torus); it should be possible to extend the discussion to more general tori [20]. In M-theory language, we will restrict to rectangular tori and vanishing three index tensor, $A_{\mu \nu \lambda}=0$. 
Thus, in addition to the manifest $S L(d, Z)$ symmetry of SYM on $\tilde{T}^{d}$ (of which, as mentioned above, only the permutation subgroup preserves the rectangular tori which we will consider), we would like the theory that underlies $d+1$ dimensional SYM to be invariant under (1.4). The group generated by permutations (the Weyl group of $S L(d, Z)$ ) and Montonen-Olive duality (1.4) is, as we will show below, the Weyl group of $E_{d}$. 3 This is the subgroup of the $\mathrm{U}$ duality group of M-theory on $T^{d}$ which takes a rectangular torus to a rectangular torus (with $A_{\mu \nu \lambda}=0$ ).

The appearance of $E_{d}$ in SYM is of course not an accident. By using the ideas of [3] one can think of SYM on $\tilde{T}^{d}$ as describing M-theory on $T^{d}$, a rectangular torus with radii $R_{1}, \cdots, R_{d}$ in the infinite momentum frame. Denoting by $R_{11}$ the radius of the longitudinal dimension, and by $l_{p}$ the eleven dimensional Planck length, the mapping between the Mtheory data $\left(R_{1}, \cdots, R_{d} ; R_{11} ; l_{p}\right)$ and the gauge theory data $\left(s_{1}, \cdots, s_{d} ; N ; g\right)$ is 1 :

$$
\begin{aligned}
s_{i} & =\frac{l_{p}^{3}}{R_{11} R_{i}} \\
g^{2} & =\frac{l_{p}^{3(d-2)}}{R_{11}^{d-3} \prod_{i=1}^{d} R_{i}}
\end{aligned}
$$

Note that the running dimensionless gauge coupling at the torus size scale,

$$
\tilde{g}^{2}=\frac{g^{2}}{\left(\prod_{i=1}^{d} s_{i}\right)^{\frac{d-3}{d}}}=\frac{l_{p}^{3}}{\left(\prod_{i=1}^{d} R_{i}\right)^{\frac{3}{d}}}
$$

is independent of $R_{11}$. As is clear from (1.6), when the volume of the M-theory torus becomes small (in eleven dimensional Planck units), the SYM becomes strongly coupled [14]. For $d<3(d>3)$ this probes the long (short) distance behavior of the theory.

Taking one of the radii $R_{1}, \cdots, R_{d}$, say $R_{k} \rightarrow 0$, we find a weakly coupled IIA string theory with coupling $g_{s}$ related to $R_{k}$ via:

$$
R_{k}=l_{s} g_{s}
$$

Here $l_{s}$ is the IIA string length which is related to $R_{k}, l_{p}$ by:

$$
\frac{1}{l_{s}^{2}}=\frac{R_{k}}{l_{p}^{3}}
$$

\footnotetext{
$3 E_{3} \equiv S L(3) \times S L(2), E_{4} \equiv S L(5), E_{5} \equiv S O(5,5), E_{6} \equiv E_{6(6)}, E_{7} \equiv E_{7(7)}, E_{8} \equiv E_{8(8)}$, $E_{9} \equiv \hat{E}_{8}$

4 ignoring factors of $2 \pi$.
} 
which is the statement that the type IIA string is the wrapped M-theory membrane. Thus the limit $R_{k} \rightarrow 0$ should be taken by sending $g_{s} \rightarrow 0$, keeping $l_{s}$ fixed (1.7), (1.8).

The resulting IIA string theory is invariant under $\mathrm{T}$ duality on the $\left(x^{i}, x^{j}\right)$ circles:

$$
R_{i} \rightarrow \frac{l_{s}^{2}}{R_{i}} ; \quad R_{j} \rightarrow \frac{l_{s}^{2}}{R_{j}} ; \quad g_{s} \rightarrow \frac{g_{s} l_{s}^{2}}{R_{i} R_{j}}
$$

T duality is a gauge symmetry (see e.g. 21] for a review) and therefore holds for finite $g_{s}$ as well. Combining it with a permutation of $R_{i} \leftrightarrow R_{j}$ one finds that (1.9) turns into the symmetry (which has been discussed in [22]):

$$
R_{i} \rightarrow \frac{l_{p}^{3}}{R_{j} R_{k}} ; \quad R_{j} \rightarrow \frac{l_{p}^{3}}{R_{i} R_{k}} ; \quad R_{k} \rightarrow \frac{l_{p}^{3}}{R_{i} R_{j}}
$$

Note in particular that it is symmetric under permutations of $i, j, k$. Translating to the SYM language by using (1.5) one finds that the $\mathrm{T}$ duality transformation (1.10) is identical to (1.4) in SYM. Thus we conclude that (1.4) has a natural M-theory interpretation as (1.10) and is a necessary property of the theory underlying SYM also from the point of view of [3]. The appearance of $E_{d}$ in SYM on $\tilde{T}^{d}$ is clearly related to the $\mathrm{U}$ duality symmetry of M-theory on $T^{d}$.

The purpose of this note is to study the algebraic structure of $\mathrm{U}$ duality for $d \geq 3$ both in the context of SYM theory and in M-theory. In section 2 we discuss "U duality" in SYM. We write down the 1/2 BPS multiplets corresponding to Kaluza-Klein modes, and to electric and magnetic fluxes. We show that permutations together with (1.4) generate the Weyl group of $E_{d}$ and exhibit directly in the SYM language the weights of $E_{d}$ corresponding to different multiplets. In the case $d=9$ we show that the multiplets correspond to representations of $\hat{E}_{8}$ at a finite level $k$ and present (weak) arguments that $k=2$.

In section 3 we discuss the interpretation of our results in M-theory. We show that wrapped D and NS branes all belong to the multiplets of section 2. Other members of these $\mathrm{U}$ duality multiplets are seen to correspond to states that in various formal weakly coupled string limits have energies that go like $1 / g_{s}^{n}$ with $n>2$. Such states occur when the number of non-compact spatial dimensions is two or less and seem to be related to a qualitative change in the physics of M-theory in low dimensions. We also show that the SYM analysis allows the existence in M-theory of a $1 / 2$ BPS eightbrane with tension $1 / l_{p}^{9}$. Such an object, if it exists, would mesh nicely with the U duality structure.

In section 4 we comment on our results; an Appendix contains a list of representations of $\mathrm{U}$ duality discussed in the text. 


\section{U duality in SYM}

\subsection{Multiplets of $U$ duality}

In SYM, "U duality" is by definition the symmetry generated by $S L(d, Z)$ and Montonen-Olive duality (1.4). In this section we will study the action of this symmetry group (or rather its subgroup generated by permutations in $S L(d, Z)$ and (1.4)) on certain $1 / 2$ BPS states of the theory.

A useful observation for the subsequent discussion is that there is a combination of $g^{2}$ and $V_{s}=\prod_{i=1}^{d} s_{i}$ that is invariant under $\mathrm{U}$ duality. The invariant combination is

$$
\frac{V_{s}^{d-5}}{g^{2(d-3)}}
$$

It scales like (energy $)^{9-d}$ and in dimensions where matrix theory is understood plays an important role. For $d=3$, the volume of the three torus is invariant under $S L(3, Z) \times$ $S L(2, Z)$. For $d=4, V_{s} g^{2}$ is invariant under $S L(5, Z)$, suggesting that $g^{2}$ should be thought of as a fifth radius [16] (see also below). For $d=5,1 / g^{2}$ is $S O(5,5 ; Z)$ invariant; this is the tension of the string that lives inside an NS fivebrane in type II string theory [19].

Beyond $d=5$ the situation is not understood. The basic invariants are:

$$
\begin{aligned}
& d=6: \frac{V_{s}}{g^{6}} \sim(\text { energy })^{3} \\
& d=7: \frac{V_{s}}{g^{4}} \sim(\text { energy }) \\
& d=8: \frac{V_{s}^{3}}{g^{10}} \sim(\text { energy }) \\
& d=9: \frac{V_{s}^{2}}{g^{6}} \sim(\text { energy })^{0}
\end{aligned}
$$

For $d<9$ the $\mathrm{U}$ duality singlets are dimensionful and thus can be set to one by performing a scale transformation. The nine dimensional case is special as the singlet is dimensionless and thus cannot be removed this way. This will play a role later.

We will discuss $1 / 2$ BPS states belonging to two U duality multiplets. The first is obtained by applying $\mathrm{U}$ duality to a Kaluza-Klein mode, carrying momentum in the $i$ 'th direction, whose energy is

$$
E_{i}^{K K}=\frac{1}{s_{i}}
$$


We will refer to this multiplet as the momentum multiplet. The second is obtained by acting on states carrying electric flux in the $i$ 'th direction:

$$
E_{i}^{E L}=\frac{g^{2} s_{i}^{2}}{N V_{s}}
$$

We will refer to it as the flux multiplet. The multiplets are generated by repeatedly applying the transformation (1.4) and permutations of the $s_{i}$ to (2.3), (2.4). The full lists of states thus obtained appear in Appendix A. Here we will describe some of their general features.

Consider first the momentum multiplet. Applying (1.4) to (2.3) gives rise to a state with energy目

$$
E=\frac{s_{j_{1}} \cdots s_{j_{d-4}}}{g^{2}}
$$

This is the Yang-Mills instanton, wrapped around $d-4$ transverse circles. It gives rise to $\left(\begin{array}{l}d \\ 4\end{array}\right)$ states. For $d=3$, (1.4) does not act on $s_{i}$, but only on $g$ (as a strong-weak coupling duality). For $d=4$, (1.4) in the directions 1,2,3 takes: $s_{4} \leftrightarrow g^{2}$, extending $S L(4)$ to $S L(5)$ and suggesting that $g^{2}$ should be thought of as a fifth radius [16]. For $d=5$, (1.4) in the directions 1,2,3 takes: $s_{4,5} \rightarrow g^{2} / s_{4,5}, g^{2} \rightarrow g^{2}$; a T duality transformation of a string with tension $g^{-2}$. For $d \geq 6$, (1.4) in the directions 1,2,3 takes a Kaluza-Klein mode $1 / s_{a}$, $a \neq 1,2,3$, to a $(d-4)$-brane with tension $g^{-2}$ wrapped on the $d-4$ directions transverse to $1,2,3, a$.

Applying (1.4) again one finds states with energy

$$
E=\frac{V_{s}}{g^{4}} \frac{s_{j_{1}} \cdots s_{j_{d-6}}}{s_{i}}
$$

Such states first appear in $d=6$ and there are $6\left(\begin{array}{l}d \\ 6\end{array}\right)$ of them. On the next level one finds states with energy

$$
E=\frac{V_{s}^{2}}{g^{6}} \frac{s_{j_{1}} \cdots s_{j_{d-7}}}{s_{i} s_{j} s_{k}}
$$

The number of such states is $35\left(\begin{array}{l}d \\ 7\end{array}\right)$.

For $d \leq 6(2.3)$, (2.5), (2.6) exhaust the states in the momentum multiplet. For $d=7,8,9$ there are additional states (2.7) and others, see Appendix A); in particular, for $d=9$ the representations are infinite. Applying permutations and (1.4) repeatedly one keeps generating new states with higher and higher powers of the dimensionless factor

5 Here and below all the indices are distinct. 
$V_{s}^{2} / g^{6}(2.2)$. The numbers of states in the representations are 3 in $d=3,5$ in $d=4,10$ in $d=5,27$ in $d=6,126$ in $d=7,2160$ in $d=8$ and $\infty$ in $d=9$. This is a sign of the $E_{d} \mathrm{U}$ duality structure. In $d=3$ the momentum multiplet is in the $(3,1)$ of $S L(3, Z) \times S L(2, Z)$, in $d=4$ it is in the 5 of $S L(5, Z)$, in $d=5$ in the 10 of $S O(5,5 ; Z)$, in $d=6$ in the 27 of $E_{6}$. For $d=7$ (as we will soon see) we find the 126 weights with $p^{2}=2$ in the adjoint (133) of $E_{7}$. For $d=8$ we find the 2160 weights with $p^{2}=4$ in the 3875 of $E_{8}$. To discuss $d=9$ we will need to understand better the $E_{9}=\hat{E}_{8}$ symmetry structure.

The same exercise can be repeated for the flux multiplet. Acting repeatedly with $U$ duality on (2.4) we find at the first few levels the following set of states:

$$
\begin{aligned}
E & =\frac{V_{s}}{N g^{2} s_{i}^{2} s_{j}^{2}} \rightarrow\left(\begin{array}{l}
d \\
2
\end{array}\right) \\
E & =\frac{V_{s} s_{i_{1}}^{2} \cdots s_{i_{d-5}}^{2}}{N g^{6}} \rightarrow\left(\begin{array}{l}
d \\
5
\end{array}\right) \\
E & =\frac{V_{s}^{3}}{N g^{10}} \frac{s_{i_{1}}^{2} \cdots s_{i_{d-7}}^{2}}{s_{i}^{2}} \rightarrow 7\left(\begin{array}{l}
d \\
7
\end{array}\right) \\
E & =\frac{V_{s}^{5}}{N g^{14}} \frac{s_{i_{1}}^{2} \cdots s_{i_{d-8}}^{2}}{s_{i}^{2} s_{j}^{2} s_{k}^{2}} \rightarrow 56\left(\begin{array}{l}
d \\
8
\end{array}\right)
\end{aligned}
$$

The first line of (2.8) corresponds to magnetic flux. The other states in (2.8) (just like (2.6), (2.7)) require better understanding 6 .

The representations of $U$ duality one finds are (see Appendix A):

$$
\begin{aligned}
& d=3:(\overline{3}, 2) \text { of } S L(3, Z) \times S L(2, Z) \\
& d=4: \overline{10} \text { of } S L(5, Z) \\
& d=5: 16 \text { of } S O(5,5 ; Z) \\
& d=6: \overline{27} \text { of } E_{6(6)}(Z) \\
& d=7: 56 \text { of } E_{7(7)}(Z) \\
& d=8: 240(\subset 248) \text { of } E_{8(8)}(Z)
\end{aligned}
$$

\subsection{The algebraic structure for $d<9$}

The way we have generated the $\mathrm{U}$ duality multiplets in the previous subsection is somewhat cumbersome and obscures the $U$ duality group. In this subsection we will

6 For $d=5$, there is only one state, $V_{s} / N g^{6}$, which does not correspond to a flux; it corresponds to a bound state of $N$ NS5 branes and a D5 brane in the description of [19]. Perhaps some of the states in $d>5$ also correspond to similar "bound states." 
show that the symmetry generated by permutations and Montonen-Olive duality (1.4) is the Weyl group of $E_{d}$ (the subgroup of $E_{d(d)}(Z)$ preserving the rectangular shape of the torus). This will allow us to study the representations in a more unified way, and help generalize the discussion to $d=9$. U duality in SYM acts on the $d+1$ dimensional space parametrized by $\left(s_{1}, \cdots, s_{d}, g^{2}\right)$ leaving the combination (2.1) fixed. Thus, it acts on the $d$ dimensional space defined by $V_{s}^{d-5} / g^{2(d-3)}=$ const. By rescaling $s_{i}, g$ we can set the constant to one (for $d \neq 9$ ) which we will do from now on:

$$
g^{2(d-3)}=V_{s}^{d-5}
$$

The $\mathrm{U}$ duality group is generated by the permutations:

$$
P_{i}: s_{i} \leftrightarrow s_{i+1} ; i=1, \cdots, d-1
$$

and (1.4). Both can be realized as reflections. Define:

$$
x_{i}=\log s_{i} ; i=1, \cdots, d
$$

Then one can think of $(2.11)$ as reflections of $d$ dimensional vectors $v$ in a hyperplane perpendicular to a given vector $\alpha$ :

$$
\sigma_{\alpha}(v)=v-2\left(\frac{v \cdot \alpha}{\alpha^{2}}\right) \alpha
$$

$P_{i}(2.11)$ corresponds to the reflection $\sigma_{\alpha_{i}}(v)$ with

$$
\alpha_{i}=e_{i+1}-e_{i} ; \quad i=1, \cdots, d-1
$$

$e_{i}$ is a unit vector in the $i$ 'th direction. One can similarly think of (1.4) as a reflection (2.13). The vector that goes to minus itself is in this case $g^{2} / W$ (see (1.1), (1.2)) or, using (2.10) $V_{s}^{\frac{d-5}{d-3}} / W$. Thus (1.4) with, say $i, j, k=1,2,3$, may be represented as a reflection $\sigma_{\alpha_{d}}$, with:

$$
\alpha_{d}=\frac{1}{d-3}\left[(d-5)\left(e_{1}+e_{2}+e_{3}\right)-2 \sum_{i=4}^{d} e_{i}\right]
$$

provided an appropriate definition of the scalar product in (2.13) is adopted. Indeed, according to (1.4) the vectors $e_{1}, e_{2}, e_{3}$ are invariant under this transformation. If it is 
a reflection $\sigma_{\alpha_{d}}$ then the scalar product in (2.13) has to be chosen such that $e_{1}$, say, is orthogonal to $\alpha_{d}$. Let us modify the usual metric by defining:

$$
e_{i} \cdot e_{j}=\delta_{i j}+\beta
$$

Notice that this modification does not change any scalar product involving the vectors $\alpha_{i}$, $i=1, \ldots, d-1$. Requiring $e_{1} \cdot \alpha_{d}$ to vanish in this metric leads to: $\beta[3(d-5)-2(d-3)]+$ $(d-5)=0$, i.e. $\beta=(d-5) /(9-d)$. The modified metric is then

$$
e_{i} \cdot e_{j}= \begin{cases}\frac{4}{9-d} & i=j \\ \frac{d-5}{9-d} & i \neq j\end{cases}
$$

It is easy to check that with the scalar product (2.17), the transformation (1.4) is indeed $\sigma_{\alpha_{d}}$ in the sense of (2.13). Furthermore, with this metric the scalar products of the $\alpha_{i}$, $i=1, \ldots, d$, are those appropriate for the simple roots of $E_{d}$ :

$$
\begin{aligned}
\alpha_{i} \cdot \alpha_{i} & =2 ; i=1, \cdots, d \\
\alpha_{i} \cdot \alpha_{i+1} & =-1 ; i=1, \cdots, d-2 \\
\alpha_{d} \cdot \alpha_{3} & =-1
\end{aligned}
$$

with all other scalar products vanishing.

To find the representations of $E_{d}(2.3),(2.5)$ - (2.7) and (2.4), (2.8) (and more generally to reproduce the results of Appendix A), one rewrites the energies of the states as $E=\exp (v \cdot x) ; v$ is the weight vector of $E_{d}$ and (2.13) acts on $v$ as the Weyl group of $E_{d}$. The momentum states (2.3) correspond to the weight vectors

$$
v_{i}^{(1)}=-e_{i}
$$

while the electric fluxes (2.4) correspond (using (2.10)) to:

$$
v_{i}^{(2)}=2 e_{i}-\frac{2}{d-3} \sum_{j=1}^{d} e_{j}
$$

The highest weight vector in the momentum representation is $\lambda_{1}=v_{1}^{(1)}=-e_{1}$. It satisfies

$$
\lambda_{1} \cdot \alpha_{i}=\delta_{i, 1}
$$


i.e. it is the fundamental weight dual to $\alpha_{1}$. The length of $\lambda_{1}$ is

$$
\lambda_{1}^{2}=\frac{4}{9-d}
$$

the correct answer from group theory.

The highest weight in the flux representation $(2.20)$ is:

$$
\lambda_{2}=v_{d}^{(2)}=2 e_{d}-\frac{2}{d-3} \sum_{i=1}^{d} e_{i}
$$

It satisfies

$$
\lambda_{2} \cdot \alpha_{i}=2 \delta_{i, d-1}
$$

and is therefore twice the fundamental weight dual to $\alpha_{d-1}$. The factor of two has a natural interpretation in M-theory, which will be mentioned in the next section. The length of $\lambda_{2}$ is

$$
\left(\frac{1}{2} \lambda_{2}\right)^{2}=\frac{10-d}{9-d}
$$

as expected from group theory.

As is clear from the formulae (2.17), (2.22), 2.25), $d=9$ is a special case that should be considered separately. We can no longer reduce the ten dimensional space parametrized by $\left(s_{1}, \cdots, s_{9}, g\right)$ to nine dimensions by requiring (2.10), and have to work in the full $9+1$ dimensional space. This is what we turn to next.

\subsection{The algebraic structure for $d=9$}

Generalizing (2.12) we define

$$
\begin{aligned}
& x_{i}=\log s_{i} \\
& x_{0}=\log g
\end{aligned}
$$

We will see that it is natural to think of the space parametrized by $\left(x_{0}, x_{1}, \cdots, x_{9}\right)$ as having metric

$$
\eta=\operatorname{diag}(-1,1,1, \cdots, 1)
$$

Thus, $x_{0}$ is timelike while $x_{i}, 1 \leq i \leq 9$, are spacelike.

The invariant for $d=9, V_{s} / g^{3}$ (2.2) corresponds to the vector

$$
d=3 e_{0}-\sum_{i=1}^{9} e_{i}
$$


Note that $d$ is null, $d^{2}=0$. The $\mathrm{U}$ duality group is generated by reflections (2.13) corresponding to the eight vectors $\alpha_{i}, i=1, \cdots, 8$ (2.14), and a ninth vector corresponding to (1.4) which is as usual (1.1), (1.2) $g^{2} / W$, or:

$$
\alpha_{9}=2 e_{0}-\sum_{i=4}^{9} e_{i}
$$

In this case the flat metric (2.27) suffices: $\alpha_{i}^{2}=2, i=1, \cdots, 9$, and the scalar products of the $\alpha_{i}$ are those of the $E_{9}$ Dynkin diagram: $\alpha_{i} \cdot \alpha_{i+1}=-1, i=1, \cdots, 7 ; \alpha_{9} \cdot \alpha_{3}=-1$, with all other scalar products vanishing.

The U duality group we see is the Weyl group of $E_{9}=\hat{E}_{8} . d$ is the null vector invariant under the Weyl group. To establish the connection with $\hat{E}_{8}$ we recall a few facts about affine Lie algebra and the action of the Weyl group (see e.g. [23]).

Consider a rank $r$ Lie algebra $G$. Representations of the affine Lie algebra $\hat{G}$ can be described in an $r+2$ dimensional space with signature $(1, r+1)$. A convenient basis is one where two weights $m=\left(\vec{\mu}, \mu_{k}, \mu_{d}\right)$ and $m^{\prime}=\left(\vec{\mu}^{\prime}, \mu_{k}^{\prime}, \mu_{d}^{\prime}\right)$ have scalar product

$$
m \cdot m^{\prime}=\vec{\mu} \cdot \vec{\mu}^{\prime}+\mu_{k} \mu_{d}^{\prime}+\mu_{d} \mu_{k}^{\prime}
$$

$\vec{\mu}$ and $\vec{\mu}^{\prime}$ belong to the weight lattice of $G$. The roots of $\hat{G}, E_{n}^{\alpha}, H_{n}^{i}(i=1, \cdots, r)$ correspond to:

$$
\begin{aligned}
& E_{n}^{\alpha} \leftrightarrow a=(\alpha, 0, n) \\
& H_{n}^{i} \leftrightarrow n \delta=(0,0, n)
\end{aligned}
$$

The simple roots of $\hat{G}$ can be chosen to be

$$
\begin{gathered}
a_{i}=\left(\beta_{i}, 0,0\right) \\
a_{0}=(-\psi, 0,1)
\end{gathered}
$$

where $\beta_{i}$ are the simple roots of $G$ and $\psi$ is the highest root of $G$.

The Weyl group of $\hat{G}$ is the group generated by reflections in hyperplanes normal to the roots (2.31). Clearly, we can only use reflections in spacelike roots $a=(\alpha, 0, n)$ since otherwise (2.13) is ill defined. All such reflections preserve $\delta$ :

$$
\sigma_{a}(\delta)=\delta
$$

Thus the Weyl group permutes space-like roots. Given two spacelike roots $a=(\alpha, 0, n)$ and $a^{\prime}=\left(\alpha^{\prime}, 0, n^{\prime}\right)$

$$
\sigma_{a}\left(a^{\prime}\right)=\left(\sigma_{\alpha}\left(\alpha^{\prime}\right), 0, n-\frac{2 \alpha \cdot \alpha^{\prime}}{\alpha^{2}} n^{\prime}\right)
$$


In our system, $G=E_{8}$, and $\delta$ is a vector proportional to $d$ (2.28). To map our $\alpha_{1}, \cdots, \alpha_{9}$ (2.14), (2.29) to the simple roots (2.32), we will assume that the roots $\alpha_{1}, \cdots, \alpha_{7}, \alpha_{9}$, whose scalar products define the Cartan matrix of $E_{8}$, correspond to the eight simple roots of $E_{8}$,

$$
\left\{\alpha_{1}, \cdots, \alpha_{7}, \alpha_{9}\right\} \leftrightarrow\left\{a_{i}=\left(\beta_{i}, 0,0\right) ; i=1, \cdots, 8\right\}
$$

In particular, we assume that they have vanishing components in the $k, d$ directions. A simple computation then leads to the conclusion that

$$
\alpha_{8}=-(\psi, 0,0)+2 d
$$

$d$ is proportional to $\delta$,

$$
\delta=n d
$$

The proportionality constant $n$ is undetermined at this level. Substituting (2.36) in (2.35) we can write $\alpha_{8}$ as:

$$
\alpha_{8}=\left(-\psi, 0, \frac{2}{n}\right)
$$

Comparing to (2.32) we see that $\alpha_{8}$ differs from $a_{0}$ by $\left(\frac{2}{n}-1\right) \delta$.

Turning to representations of $E_{9}$, the highest weight vectors corresponding to (2.3), (2.4) are:

$$
\begin{aligned}
& \lambda_{1}=-e_{1} \\
& \lambda_{2}=2 e_{0}+e_{9}-\sum_{i=1}^{8} e_{i}
\end{aligned}
$$

$\lambda_{1}$ is the fundamental weight dual to $\alpha_{1}$ (the 3875 of $E_{8}$ ); $\lambda_{2}$ is twice the fundamental weight corresponding to $\alpha_{8}$ (the current algebra block of the identity). The level $k$ of the $\hat{E}_{8}$ represented by $(2.38)$ is obtained by evaluating $\lambda_{i} \cdot \delta=\lambda_{i} \cdot n d=n$. Thus the level of $\hat{E}_{8}$ is:

$$
k=n
$$

Note that the fact that one gets the same level $k$ for the two representations (2.38) is due to the fact that $\lambda \cdot \delta$ measures the scaling dimension of $\exp (\lambda \cdot x)$. Any object constructed out of $g, s_{i}$ that scales like energy will give rise to the same level (2.39). In the basis (2.31) we have:

$$
\begin{aligned}
& \lambda_{1}=\left(\mu_{1}, n, a_{1}\right) \\
& \lambda_{2}=\left(0, n, a_{2}\right)
\end{aligned}
$$


where $\mu_{1}^{2}=4$ and $a_{1}, a_{2}$ can be determined by requiring that $\lambda_{1}^{2}=1, \lambda_{2}^{2}=5$, which leads to $a_{1}=-\frac{3}{2 n}, a_{2}=\frac{5}{2 n}$. The vector $k=(0,1,0)$ is given by:

$$
k=\frac{1}{n}\left(-\frac{11}{2} e_{0}+\frac{7}{2} e_{9}+\frac{3}{2} \sum_{i=1}^{8} e_{i}\right)
$$

At this point, the level of the $\hat{E}_{8}(2.39)$ remains undetermined. Since reflections by $\alpha_{8}$ will change the value of $L_{0}$ (the descendant level) by $2 / n$, and one expects differences of $L_{0}$ to be integer, it appears that $n \leq 2$. Furthermore, if $n=1$, then some of the states would have energies that are odd powers of the SYM coupling $g$. Since odd powers of $g$ are not expected to appear in this case, the most natural choice appears to be $k=n=2$; however a more careful analysis is necessary here.

\section{M-theory interpretation}

By using the mapping between the M-theory variables and those of SYM, we can translate the spectra obtained in the previous section to the language of M-theory on $T^{d}$. Recall [3] that the SYM energy is interpreted in matrix theory as $P^{-}=E-p_{11}$. States with momentum $p_{11}=N / R_{11}$ and mass $M$ satisfy $E=\sqrt{p_{11}^{2}+M^{2}}$. If $p_{11}>>$ one has $E \simeq p_{11}+M^{2} / 2 p_{11}$ or $P^{-}=M^{2} / P^{+}$. Thus the SYM energy and the M-theory mass are related viat:

$$
M=\sqrt{E_{S Y M} \frac{N}{R_{11}}}
$$

For objects that wrap the eleventh direction, the relation between $M$ and $E_{S Y M}$ is linear

$$
M=E_{S Y M}
$$

The full results for the mapping of SYM to M-theory are in Appendix A. Here we will describe the structure of the states $(2.3)-(2.8)$ mentioned in section 2 .

The momentum multiplet, (2.3), 2.5 - (2.7), describes branes wrapped around the eleventh dimension. In particular

$$
\begin{aligned}
& E=\frac{1}{s_{i}} \leftrightarrow M=\frac{R_{11} R_{i}}{l_{p}^{3}} \\
& E=\frac{s_{i_{1}} \cdots s_{i_{d-4}}}{g^{2}} \leftrightarrow M=\frac{R_{11} R_{j_{1}} \cdots R_{j_{4}}}{l_{p}^{6}} \\
& E=\frac{V_{s}}{g^{4}} \frac{s_{i_{1}} \cdots s_{i_{d-6}}}{s_{i}} \leftrightarrow M=\frac{R_{11} R_{i}^{2} R_{j_{1}} \cdots R_{j_{5}}}{l_{p}^{9}} \\
& E=\frac{V_{s}^{2}}{g^{6}} \frac{s_{i_{1}} \cdots s_{i_{d-7}}}{s_{i} s_{j} s_{k}} \leftrightarrow M=\frac{R_{11} R_{i}^{2} R_{j}^{2} R_{k}^{2} R_{j_{1}} \cdots R_{j_{4}}}{l_{p}^{12}}
\end{aligned}
$$

7 We ignore factors of 2. 
The Kaluza-Klein modes correspond in M-theory to wrapped membranes, SYM instantons give rise to wrapped fivebranes. The other two objects are more exotic; we will return to the first of them later.

Moving on to the flux multiplet, one finds:

$$
\begin{gathered}
E=\frac{g^{2} s_{i}^{2}}{N V_{s}} \leftrightarrow M=\frac{1}{R_{i}} \\
E=\frac{V_{s}}{N g^{2} s_{i}^{2} s_{j}^{2}} \leftrightarrow M=\frac{R_{i} R_{j}}{l_{p}^{3}} \\
E=\frac{V_{s} s_{i_{1}}^{2} \cdots s_{i_{d-5}}^{2}}{N g^{6}} \leftrightarrow M=\frac{R_{j_{1}} \cdots R_{j_{5}}}{l_{p}^{6}} \\
E=\frac{V_{s}^{3}}{N g^{10}} \frac{s_{i_{1}}^{2} \cdots s_{i_{d-7}}^{2}}{s_{i}^{2}} \leftrightarrow M=\frac{R_{i}^{2} R_{j_{1}} \cdots R_{j_{6}}}{l_{p}^{9}} \\
E=\frac{V_{s}^{5}}{N g^{14}} \frac{s_{i_{1}}^{2} \cdots s_{i_{d-8}}^{2}}{s_{i}^{2} s_{j}^{2} s_{k}^{2}} \leftrightarrow M=\frac{R_{i}^{2} R_{j}^{2} R_{k}^{2} R_{j_{1}} \cdots R_{j_{5}}}{l_{p}^{12}}
\end{gathered}
$$

Thus states carrying electric flux describe Kaluza-Klein modes in eleven dimensions, states carrying magnetic flux correspond to wrapped membranes, etc. We see that the states one finds in the two multiplets are similar except for the wrapping around $x^{11}$. Note also that the translation to M-theory provides a natural explanation for why the highest weight $\lambda_{1}$ corresponding to the momentum multiplet is equal to a fundamental weight, while $\lambda_{2}$, which corresponds to the flux multiplet is equal to twice a fundamental weight. In the translation to M-theory, SYM energies in the momentum multiplet are translated to masses, while those in the flux multiplet are translated to masses squared.

At this point we still face two puzzles:

(a) How should one regulate SYM to find states with the energies listed in Appendix A?

(b) What is the meaning of the states one finds in M-theory?

We will leave the first question to future work, and discuss briefly a puzzling aspect of the second one, having to do with the behavior of some of the masses in various weakly coupled string limits. Recall that to consider weakly coupled strings we take one of the radii and $l_{p}$ to zero (1.7) keeping the string length $l_{s}$ fixed: $R=l_{s} g_{s}, l_{p}^{3}=l_{s}^{3} g_{s}, g_{s} \rightarrow 0$. States in M-theory whose energy diverges like $1 / l_{p}^{9}$ or faster in the limit, potentially have masses that go like $1 / g_{s}^{n}$ with $n>2$, which is surprising in a weakly coupled string theory, where the most singular behavior that is expected is $1 / g_{s}^{2}$ (corresponding to solitons). 
The simplest states with mass that diverges more rapidly have 8

$$
M=\frac{R_{1}^{2} R_{2} \cdots R_{7}}{l_{p}^{9}}
$$

(see (3.3), (3.4)). These states describe a number of different objects. If we choose $R_{1}$ to correspond to $g_{s}$, they become Dirichlet sixbranes wrapped around $x_{2}, \cdots, x_{7}$ with mass

$$
M=\frac{R_{2} \cdots R_{7}}{g_{s} l_{s}^{7}}
$$

If we choose (say) $R_{7}$ to correspond to $g_{s}$ we get a solitonic object with mass

$$
M=\frac{R_{1}^{2} R_{2} \cdots R_{6}}{g_{s}^{2} l_{s}^{8}}
$$

which can be thought of as the T dual of the NS fivebrane on one circle inside its worldvolume and one circle transverse to it $\mathrm{Q}$. If we shrink a circle that is not in the list $\left(x_{1}, \cdots, x_{7}\right)$, say $x_{8}$, we find an object with mass

$$
M=\frac{R_{1}^{2} R_{2} \cdots R_{7}}{g_{s}^{3} l_{s}^{9}}
$$

which diverges more rapidly than that of a soliton. One way of thinking about it is by starting with a Dirichlet sevenbrane of type IIB string theory (the $(1,0)$ sevenbrane), performing an $S L(2, Z)$ transformation on it, turning it into a $(0,1)$ sevenbrane, and then a further $\mathrm{T}$ duality on one circle to pass to a type IIA picture. The $(0,1)$ sevenbrane is a rather singular object; there is no sensible weak coupling expansion for a fundamental type IIB string in its presence.

A more direct way to observe that weak coupling is not valid for states such as (3.6) is to consider the gravitational strength of such an object. The gravitational strength is proportional to both the mass $M$ of the object and to Newton's constant $G$. The latter is proportional to $g_{s}^{2}$. Thus for objects whose mass is proportional to $1 / g_{s}^{a}$ with $a<2$, the gravitational strength vanishes in the $g_{s} \rightarrow 0$ limit allowing for an asymptotic flat space even in their presence. For $a>2$ a very large gravitational field is created by these objects in the supposedly weak coupling limit. Thus weak coupling cannot be trusted. What the presence of such objects implies for the structure of compactified M-theory,

8 These are identified in M-theory with KK monopoles, which are 6-branes with tension $R_{i}^{2} / l_{p}^{9}$.

9 This is a KK monopole in type II string, which is a 5-brane with tension $R_{i}^{2} / g_{s}^{2} l_{s}^{8}$. 
and in particular for the fate of space-time when there are fewer than $3+1$ non-compact directions, remains to be understood.

As is clear from Appendix A, there are many 1/2 BPS states with singular energies. In type II string theory on $T^{7}$ (M-theory on $T^{8}$ ) we find states with $M \sim \frac{1}{g_{s}^{n}}$ with $n=3,4$, while on $T^{8}$ (M-theory on $T^{9}$ ) $n$ is not bounded. It would be interesting to understand what are the implications of all these states for low dimensional string (M-) theory.

It is easy to identify in the momentum and flux multiplets states which correspond to all possible wrapped D0, D2, D4, D6, D8 and NS5 branes of the type IIA string. For instance, wrapped D 8 branes appear in the $d=8$ momentum multiplet, corresponding to SYM states with energy $E=V_{s}^{2} /\left(g^{6} s_{i}^{2}\right)$; choosing $R_{i}=l_{s} g_{s}$ one finds a D8 brane wrapped on $R_{11}$ and seven other directions. Wrapped D8 branes also appear in the $d=9$ flux multiplet (see Appendix A), corresponding to SYM states with energy $E=V_{s}^{5} /\left(N g^{14} s_{i}^{4}\right)$; choosing $R_{i}=l_{s} g_{s}$ one finds a D8 brane wrapped on eight transverse circles.

One can also try to "work backwards" from M-theory, and ask which other branes in M-theory are consistent with the SYM description. A wrapped M-theory $p$-brane has a mass

$$
M=R_{11} R_{i_{1}} \cdots R_{i_{p-1}} / l_{p}^{p+1}
$$

if it wraps $x^{11}$, or

$$
M=R_{i_{1}} \cdots R_{i_{p}} / l_{p}^{p+1}
$$

if it wraps $p$ transverse circles. Translating (3.7), (3.8) to SYM variables (1.5), we find SYM states with energies:

$$
\begin{gathered}
E=\left(\frac{V_{s}}{g^{2}}\right)^{\frac{p-2}{3}} \frac{1}{s_{i_{1}} \cdots s_{i_{p-1}}} \\
E=\frac{1}{N}\left(\frac{V_{s}}{g^{2}}\right)^{\frac{2 p-1}{3}} \frac{1}{s_{i_{1}}^{2} \cdots s_{i_{p}}^{2}}
\end{gathered}
$$

Wrapped BPS saturated branes should correspond to integer powers $\mathbb{E}$ of $g^{2}$, $V_{s}$, i.e. to $p=2 \bmod 3$. We have already seen the M-theory membrane, $p=2$, corresponding to SYM

10 Note that states whose energies are proportional to non-integer powers of $g^{2}, V_{s}$ certainly exist in the spectrum, but they are not BPS saturated. E.g. states corresponding to small membranes or fivebranes of size $\simeq l_{p}$ have energies $E \sim 1 / l_{p}$, corresponding to (3.8) with $p=0$. In SYM, (3.10), their energies go like $E \sim\left(\frac{g^{2}}{V_{s}}\right)^{\frac{1}{3}}$; these are the familiar Supersymmetric Quantum Mechanics excitations with energy $E \sim g_{S Q M}^{\frac{2}{3}}[24,25,26]$. 
momenta and magnetic fluxes, and fivebrane, $p=5$, corresponding to SYM instantons in the momentum multiplet, and states at the third level of the flux multiplet (3.3), (3.4).

Equations (3.9), (3.10) seem to also allow an eightbrane with tension $T \simeq 1 / l_{p}^{9}$. Such an object is not known to exist in M-theory, but appears to mesh nicely with the algebraic structure described in section 2. For $d=7$ the only finite energy state of a wrapped eightbrane corresponds to one wrapped around the seven torus and $x^{11}$. Its SYM energy (3.9) is $E=V_{s} / g^{4}$, which is the singlet of $E_{7}$ encountered in section 2 (2.2). In $d=8$ we can wrap the eightbrane around a transverse eight torus, with SYM energy (3.10) $E=V_{s}^{3} / \mathrm{Ng}^{10}$. Again, this corresponds to a singlet of $E_{8}$ (2.2). Alternatively, one can consider a longitudinal eightbrane, wrapping a seven torus and $x^{11}$. The SYM energy is in this case (3.9) $E=V_{s} s_{i} / g^{4}$. Repeating the analysis in section 2 leads to the highest weight

$$
\lambda_{3}=e_{8}-\frac{1}{5} \sum_{i=1}^{8} e_{i}
$$

which is the fundamental weight dual to $\alpha_{7}=e_{8}-e_{7}$ (the adjoint of $E_{8}$ ). In particular, comparing to (2.23) we see that $\lambda_{3}=\lambda_{2} / 2$.

In $d=9$, the longitudinal eightbrane (3.9) has SYM energy $E=V_{s} s_{i} s_{j} / g^{4}$ while the transverse one (3.10) has energy $E=V_{s}^{3} s_{i}^{2} / N g^{10}$. The corresponding highest weights are:

$$
\begin{aligned}
& \lambda_{3}=e_{8}+e_{9}+\sum_{i=1}^{9} e_{i}-4 e_{0}=\left(\mu_{3}, n,-\frac{3}{2 n}\right) \\
& \lambda_{4}=2 e_{9}+3 \sum_{i=1}^{9} e_{i}-10 e_{0}=\left(0, n,-\frac{3}{2 n}\right)
\end{aligned}
$$

where $\mu_{3}$ is the fundamental weight dual to $\alpha_{7}$, corresponding to the adjoint of $E_{8}$ (i.e. $\mu_{3}=\psi$, the highest root). Thus, the longitudinal wrapped eightbrane gives rise to the only other representation of a unitary level $2 \hat{E}_{8}$ affine Lie algebra not seen before (2.38). The transverse eightbrane $\lambda_{4}$ gives rise to states in the current algebra block of the identity, hence it belongs to the same multiplet as $\lambda_{2}$ (2.38).

It is not clear, despite the algebraic appeal, whether a $1 / 2$ BPS eightbrane with tension $\simeq 1 / l_{p}^{9}$ indeed exists in M-theory. There is in principle no reason to expect unitary representations of $\hat{E}_{8}$ to occur in this problem as the $\hat{E}_{8}$ symmetry is non-compact. In any case, upon reduction to type IIA string theory, one would expect such an eightbrane to give rise to a seven brane with tension $\simeq 1 / g_{s}^{2} l_{s}^{8}$, and to an eightbrane with tension 
$\simeq 1 / g_{s}^{3} l_{s}^{9}$. These high dimensional objects are plagued by IR subtleties, and their role in the dynamics is unclear, much like that of other heavy high dimensional objects mentioned above.

\section{Discussion}

We end with a few comments on our results.

1) When $R_{11}$ is finite, what we have called M-theory on $T^{d}$ is really M-theory on $T^{d+1}$. The IMF description of M-theory provided by the SYM construction should hold also in the limit $N \rightarrow \infty, R_{11}$ finite. In that limit we should be seeing a larger $\mathrm{U}$ duality group, $E_{d+1}$. Since $R_{11}$ and the transverse radii are treated differently in the SYM description, it is not as easy to see this symmetry enhancement. The larger symmetry combines the momentum and flux multiplets discussed above into a single multiplet of $E_{d+1}$. It would be interesting to understand the action of $E_{d+1}$ directly on the SYM variables 11 ; this will involve achieving a better understanding of rotational invariance of the theory.

2) An important question is what is the theory that underlies SYM and exhibits the $E_{d}$ symmetry discussed above. How much of the high energy behavior of the theory is actually necessary for understanding the spectra (3.3), (3.4)? Most of the states in Appendix A have energies that go like high powers of $g^{-2}$, which seems to suggest that they are sensitive to very high energy physics. On the other hand, we have obtained them by applying Montonen-Olive duality to conventional SYM states which seems to suggest that one can hope to understand them in a regulated version of SYM with few additional degrees of freedom.

An example of such a state is the transverse fivebrane, which proved elusive in SQM [28], and whose full understanding seems to require a 5+1 dimensional string theory [19]. In our discussion, the transverse fivebrane corresponded to a SYM state (third line of (3.4)) with $E \simeq 1 / g^{6}$, which was obtained from a state carrying magnetic flux (second line of (3.4) ) by applying Montonen-Olive duality. The relative $1 / g^{4}$ factor in the energies of the two objects seems to suggest that the fivebrane corresponds to a state with strong SYM fields, for which the short distance structure of the underlying theory is important. On the other hand, such relative factors of $1 / g^{4}$ must already occur in $3+1$ dimensional

11 In a recent paper [27] it was suggested that Nahm type transformations implement rotations of $x^{11}$ into $x^{i}$ in Matrix theory. 
SYM. Indeed, consider 12 a small (unwrapped, non BPS) membrane in M-theory on $T^{3}$. It has $E \simeq 1 / l_{p}$ and as discussed in section 3, (3.7) - (3.10) corresponds to a SYM state with $E_{2} \simeq \frac{1}{N}\left(\frac{g^{2}}{V_{s}}\right)^{\frac{1}{3}}$ (which can be studied in SQM [3, 24, 225,26]). In M-theory on $T^{3}$ the unwrapped membrane is dual (under $S L(2, Z)$ ) to a fivebrane wrapped on $T^{3}$ [22], which by (3.10) has energy $E_{5} \simeq\left(1 / g^{4}\right) E_{2}$. Thus, Montonen-Olive duality in $3+1$ dimensional SYM must take rather standard states to more exotic states with singular energies. Understanding how this occurs may help understand the transverse fivebrane in higher dimensional SYM.

3) The occurrence of states with very singular masses in "weak coupling" regions seems to suggest a qualitative change in the physics of M-theory below $3+1$ non-compact dimensions (see also [29]). There have been proposals that quantum mechanically space-time disappears in these low dimensions. It is important to clarify the structure of low dimensional M-theory especially since, as discussed in the introduction, it may exhibit the full algebraic structure of the underlying theory. In this context it should be mentioned that a recent proposal [30] relates M-theory on $T^{9}$ to $N=(2,1)$ heterotic strings [31].

4) By the time one has studied M-theory compactified on a nine-torus, the original cast of characters has grown significantly. It seems appropriate to reexamine what has been achieved. A regularization of many low energy higher dimensional field theories is achieved already in string theory itself. This regularization is however perturbative in nature. By now it has been shown that the Matrix approach indeed seems to give a rather direct access to various nonperturbative symmetries, continuous versions of which have been observed long ago in low energy supergravity theories. There are many other reasons to believe that string theory has a rich nonperturbative structure. Not the least of these hints is the fact that string theory has a Hagedorn limiting temperature. It is tempting to identify it as a phase transition to new, more "fundamental" degrees of freedom. Leaving aside possible inherent difficulties in defining the concept of temperature in a system containing quantum gravity, one could ask what do Matrix models suggest for the density of states at high temperature. Evaluating that, one needs to recall that we sit in the light front where one is calculating $\operatorname{Tr} \exp \left(-\beta P^{-}\right)$. For $d=1$ the Hagedorn density is recaptured signaling that from that point of view D0 branes are indeed a simplification. However, already for $1<d \leq 3$, what seems like a well behaved SYM gauge theory in the light front, actually disguises a density of states as a function of their mass diverging even faster than

12 We thank S. Shenker for a discussion on this issue. 
the Hagedorn density of states. For $3<d \leq 9$ the trend is only reinforced. It seems that the fundamental theory acquires more and more the form of the original M-theory. In a sense one is dragged to be doing M-theory for M-theory.

5) A related analysis of the U duality group in supergravity was recently performed in [32].

Acknowledgements: We thank I. Bakas, M. Bershadsky, E. Kiritsis, M. Green, E. Martinec, G. Moore and S. Shenker for discussions. This work is supported in part by the Israel Academy of Sciences and Humanities - Centers of Excellence Program. The work of A. G. and E. R. is supported in part by BSF - American-Israel Bi-National Science Foundation. S. E., A. G. and E. R. thank the Einstein Center at the Weizmann Institute for partial support. D. K. is supported in part by a DOE OJI grant and thanks the Aspen Center for Physics for hospitality. A. G. thanks the Theory Division at CERN for hospitality.

Note Added: The 8-brane with tension $1 / l_{p}^{9}$, discussed in section 3, naturally completes the momentum and flux multiplets into $E_{d}$ representations. The 126, 240 and 2160 states in tables 10, 11 and 12, respectively, correspond to the long weights in the representations 133, 248 and 3875. The shorter weights in these $E_{7}$ and $E_{8}$ representations correspond to entries in the tables above that contain ratios $\left(R_{i} / R_{j}\right)$ with $i=j$. These correspond to states in the singlet or $\mathbf{2 4 8}$ multiplets of the transverse or longitudinal 8-brane, discussed in the text, and appear with the right multiplicity to fill out the $E_{7}$ and $E_{8}$ representations. Explicitly, in the third row of table 10 there are states with energies $\left(V_{R} R_{11} / l_{p}^{9}\right)\left(R_{i} / R_{j}\right)$ which for $i=j, i=1, \ldots, 7$ give rise to the longitudinal 8-brane with multiplicity 7 corresponding to the CSA of $E_{7}$. Similarly, in the fourth row of table 11 there are states with energies $\left(V_{R} / l_{p}^{9}\right)\left(R_{i} / R_{j}\right)$ which for $i=j, i=1, \ldots, 8$ give rise to the transverse 8 -brane with multiplicity 8 corresponding to the CSA of $E_{8}$. Using the same ansatz for the states in rows $3,4,6,8,10,12,13$ of table 12 we find $7 \times 240+35 \times 1$ states required to fill out the 3875 of $E_{8}$. The 240 is the multiplet obtained from longitudinal 8-branes by acting with U-duality; the 35 singlets are different states with energy $\left.V_{R}^{2} R_{11} / l_{p}^{18}\right)$.

It is an interesting open problem to find both the eightbranes and the additional states in supergravity and in particular to explain their degeneracy. Moreover, it is interesting that one seems to be led to highest weight representations of the full $E_{d}$ group, despite the fact that the actual U-duality symmetry is a discrete non-compact version of $E_{d}$. 


\section{Appendix A. U duality multiplets in SYM and M-theory}

In this appendix we collect some results on the $\mathrm{U}$ duality multiplet corresponding to Kaluza-Klein modes, and the multiplet corresponding to electric and magnetic fluxes in SYM. The masses $M$ in M-theory are related to the energies $E_{S Y M}=P^{-}$in the SYM theory as follows:

$S Y M$ fluxes multiplet $\left(P^{+}=\frac{N}{R_{11}}\right)$ :

$$
P^{-}=\frac{M^{2}}{P^{+}}
$$

SYM momentum multiplet:

$$
P^{-}=M
$$

In the tables below, states are labeled by indices all of which are different. We present the decomposition of the $E_{d}$ U-multiplets into $S L(d)$ representations. Recall that

$$
V_{s}=\prod_{i=1}^{d} s_{i}, \quad V_{R}=\prod_{i=1}^{d} R_{i}
$$

The fact that we study orbits of the Weyl group implies that we obtain only the nonzero roots of the adjoint representation of $E_{7}, E_{8}$, and the weights with length ${ }^{2}=4$ of the 3875 in $E_{8}$. For $d=9$ the representations are infinite; we write down a few of the low lying states in the $\hat{E}_{8}$ Weyl trajectories. The table captions contain the total number of states in the representations. 


\begin{tabular}{|c|c|c|}
\hline$S L(3)$ & $E_{S Y M}$ & $M$ \\
\hline 3 & $\frac{g^{2} s_{i}^{2}}{N V_{s}}$ & $\frac{1}{R_{i}}$ \\
\hline 3 & $\frac{V_{s}}{N g^{2} s_{i}^{2} s_{j}^{2}}$ & $\frac{R_{i} R_{j}}{l_{p}^{3}}$ \\
\hline
\end{tabular}

Table 1: $d=3$, fluxes multiplet: $3+3$

\begin{tabular}{|c|c|c|}
\hline$S L(3)$ & $E_{S Y M}$ & $M$ \\
\hline 3 & $\frac{1}{s_{i}}$ & $\frac{R_{11} R_{i}}{l_{p}^{3}}$ \\
\hline
\end{tabular}

Table 2: $d=3$, momentum multiplet: 3

\begin{tabular}{|c|c|c|}
\hline$S L(4)$ & $E_{S Y M}$ & $M$ \\
\hline 4 & $\frac{g^{2} s_{i}^{2}}{N V_{s}}$ & $\frac{1}{R_{i}}$ \\
\hline 6 & $\frac{V_{s}}{N g^{2} s_{i}^{2} s_{j}^{2}}$ & $\frac{R_{i} R_{j}}{l_{p}^{3}}$ \\
\hline
\end{tabular}

Table 3: $d=4$, fluxes multiplet: 10

\begin{tabular}{|c|c|c|}
\hline$S L(4)$ & $E_{S Y M}$ & $M$ \\
\hline 4 & $\frac{1}{s_{i}}$ & $\frac{R_{11} R_{i}}{l_{p}^{3}}$ \\
\hline 1 & $\frac{1}{g^{2}}$ & $\frac{V_{R} R_{11}}{l_{p}^{6}}$ \\
\hline
\end{tabular}

Table 4: $d=4$, momentum multiplet: 5 


\begin{tabular}{|c|c|c|}
\hline$S L(5)$ & $E_{S Y M}$ & $M$ \\
\hline 5 & $\frac{g^{2} s_{i}^{2}}{N V_{s}}$ & $\frac{1}{R_{i}}$ \\
\hline 10 & $\frac{V_{s}}{N g^{2} s_{i}^{2} s_{j}^{2}}$ & $\frac{R_{i} R_{j}}{l_{p}^{3}}$ \\
\hline 1 & $\frac{V_{s}}{N g^{6}}$ & $\frac{V_{R}}{l_{p}^{6}}$ \\
\hline
\end{tabular}

Table 5: $d=5$, fluxes multiplet: 16

\begin{tabular}{|c|c|c|}
\hline$S L(5)$ & $E_{S Y M}$ & $M$ \\
\hline 5 & $\frac{1}{s_{i}}$ & $\frac{R_{11} R_{i}}{l_{p}^{3}}$ \\
\hline 5 & $\frac{s_{i}}{g^{2}}$ & $\frac{V_{R} R_{11}}{l_{p}^{6} R_{i}}$ \\
\hline
\end{tabular}

Table 6: $d=5$, momentum multiplet: 10

\begin{tabular}{|c|c|c|}
\hline$S L(6)$ & $E_{S Y M}$ & $M$ \\
\hline 6 & $\frac{g^{2} s_{i}^{2}}{N V_{s}}$ & $\frac{1}{R_{i}}$ \\
\hline 15 & $\frac{V_{s}}{N g^{2} s_{i}^{2} s_{j}^{2}}$ & $\frac{R_{i} R_{j}}{l_{p}^{3}}$ \\
\hline 6 & $\frac{V_{s} s_{i}^{2}}{N g^{6}}$ & $\frac{V_{R}}{l_{p}^{6} R_{i}}$ \\
\hline
\end{tabular}

Table 7: $d=6$, fluxes multiplet: 27

\begin{tabular}{|c|c|c|}
\hline$S L(6)$ & $E_{S Y M}$ & $M$ \\
\hline 6 & $\frac{1}{s_{i}}$ & $\frac{R_{11} R_{i}}{l_{p}^{3}}$ \\
\hline 15 & $\frac{s_{i} s_{j}}{g^{2}}$ & $\frac{V_{R} R_{11}}{l_{p}^{6} R_{i} R_{j}}$ \\
\hline 6 & $\frac{V_{s}}{g^{4} s_{i}}$ & $\frac{V_{R} R_{11} R_{i}}{l_{p}^{9}}$ \\
\hline
\end{tabular}

Table 8: $d=6$, momentum multiplet: 27 


\begin{tabular}{|c|c|c|}
\hline$S L(7)$ & $E_{S Y M}$ & $M$ \\
\hline 7 & $\frac{g^{2} s_{i}^{2}}{N V_{s}}$ & $\frac{1}{R_{i}}$ \\
\hline 21 & $\frac{V_{s}}{N g^{2} s_{i}^{2} s_{j}^{2}}$ & $\frac{R_{i} R_{j}}{l_{p}^{3}}$ \\
\hline 21 & $\frac{V_{s} s_{i}^{2} s_{j}^{2}}{N g^{6}}$ & $\frac{V_{R}}{l_{p}^{6} R_{i} R_{j}}$ \\
\hline 7 & $\frac{V_{s}^{3}}{N g^{10} s_{i}^{2}}$ & $\frac{V_{R} R_{i}}{l_{p}^{9}}$ \\
\hline
\end{tabular}

Table 9: $d=7$, fluxes multiplet: 56

\begin{tabular}{|c|c|c|}
\hline$S L(7)$ & $E_{S Y M}$ & $M$ \\
\hline 7 & $\frac{1}{s_{i}}$ & $\frac{R_{11} R_{i}}{l_{p}^{3}}$ \\
\hline 35 & $\frac{s_{i} s_{j} s_{k}}{g^{2}}$ & $\frac{V_{R} R_{11}}{l_{p}^{6} R_{i} R_{j} R_{k}}$ \\
\hline 42 & $\frac{V_{s}}{g^{4}} \frac{s_{i}}{s_{j}}$ & $\frac{V_{R} R_{11}}{l_{p}^{9}} \frac{R_{j}}{R_{i}}$ \\
\hline 35 & $\frac{V_{s}^{2}}{g^{6} s_{i} s_{j} s_{k}}$ & $\frac{V_{R} R_{11} R_{i} R_{j} R_{k}}{l_{p}^{12}}$ \\
\hline 7 & $\frac{V_{s}^{2} s_{i}}{g^{8}}$ & $\frac{V_{R}^{2} R_{11}}{l_{p}^{15} R_{i}}$ \\
\hline
\end{tabular}

Table 10: $d=7$, momentum multiplet: $126(\subset 133)$

\begin{tabular}{|c|c|c|}
\hline$S L(8)$ & $E_{S Y M}$ & $M$ \\
\hline 8 & $\frac{g^{2} s_{i}^{2}}{N V_{s}}$ & $\frac{1}{R_{i}}$ \\
\hline 28 & $\frac{V_{s}}{N g^{2} s_{i}^{2} s_{j}^{2}}$ & $\frac{R_{i} R_{j}}{l_{p}^{3}}$ \\
\hline 56 & $\frac{V_{s} s_{i}^{2} s_{j}^{2} s_{k}^{2}}{N g^{6}}$ & $\frac{V_{R}}{l_{p}^{6} R_{i} R_{j} R_{k}}$ \\
\hline 56 & $\frac{V_{s}^{3}}{N g^{10}} \frac{s_{i}^{2}}{s_{j}^{2}}$ & $\frac{V_{R}}{l_{p}^{9}} \frac{R_{j}}{R_{i}}$ \\
\hline 56 & $\frac{V_{s}^{5}}{N g^{14} s_{i}^{2} s_{j}^{2} s_{k}^{2}}$ & $\frac{V_{R} R_{i} R_{j} R_{k}}{l_{p}^{12}}$ \\
\hline 28 & $\frac{V_{s}^{5} s_{i}^{2} s_{j}^{2}}{N g^{18}}$ & $\frac{V_{R}^{2}}{l_{p}^{15} R_{i} R_{j}}$ \\
\hline 8 & $\frac{V_{s}^{7}}{N g^{22} s_{i}^{2}}$ & $\frac{V_{R}^{2} R_{i}}{l_{p}^{18}}$ \\
\hline
\end{tabular}

Table 11: $d=8$, fluxes multiplet: $240(\subset 248)$ 


\begin{tabular}{|c|c|c|}
\hline$S L(8)$ & $E_{S Y M}$ & $M$ \\
\hline 8 & $\frac{1}{s_{i}}$ & $\frac{R_{11} R_{i}}{l_{p}^{3}}$ \\
\hline 70 & $\frac{s_{i} s_{j} s_{k} s_{l}}{g^{2}}$ & $\frac{V_{R} R_{11}}{l_{p}^{6} R_{i} R_{j} R_{k} R_{l}}$ \\
\hline 168 & $\frac{V_{s}}{g^{4}} \frac{s_{i} s_{j}}{s_{k}}$ & $\frac{V_{R} R_{11}}{l_{p}^{9}} \frac{R_{k}}{R_{i} R_{j}}$ \\
\hline 280 & $\frac{V_{s}^{2}}{g^{6}} \frac{s_{i}}{s_{j} s_{k} s_{l}}$ & $\frac{V_{R} R_{11}}{l_{p}^{12}} \frac{R_{j} R_{k} R_{l}}{R_{i}}$ \\
\hline 8 & $\frac{V_{s}^{2}}{g^{6} s_{i}^{2}}$ & $\frac{V_{R} R_{11} R_{i}^{2}}{l_{p}^{12}}$ \\
\hline 280 & $\frac{V_{s}^{2}}{g^{8}} \frac{s_{i} s_{j} s_{k} s_{l}}{s_{m}}$ & $\frac{V_{R}^{2} R_{11}}{l_{p}^{15}} \frac{R_{m}}{R_{i} R_{j} R_{k} R_{l}}$ \\
\hline 56 & $\frac{V_{s}^{2} s_{i}^{2} s_{j}}{g^{8}}$ & $\frac{V_{R}^{2} R_{11}}{l_{p}^{15} R_{i}^{2} R_{j}}$ \\
\hline 420 & $\frac{V_{s}^{3}}{g^{10}} \frac{s_{i} s_{j}}{s_{k} s_{l}}$ & $\frac{V_{R}^{2} R_{11}}{l_{p}^{18}} \frac{R_{k} R_{l}}{R_{i} R_{j}}$ \\
\hline 56 & $\frac{V_{s}^{4}}{g^{12} s_{i}^{2} s_{j}}$ & $\frac{V_{R}^{2} R_{11} R_{i}^{2} R_{j}}{l_{p}^{21}}$ \\
\hline 280 & $\frac{V_{s}^{4}}{g^{12}} \frac{s_{m}}{s_{i} s_{j} s_{k} s_{l}}$ & $\frac{V_{R}^{2} R_{11}}{l_{p}^{21}} \frac{R_{i} R_{j} R_{k} R_{l}}{R_{m}}$ \\
\hline 8 & $\frac{V_{s}^{4} s_{i}^{2}}{g^{14}}$ & $\frac{V_{R}^{3} R_{11}}{l_{p}^{24} R_{i}^{2}}$ \\
\hline 280 & $\frac{V_{s}^{4}}{g^{14}} \frac{s_{j} s_{k} s_{l}}{s_{i}}$ & $\frac{V_{R}^{3} R_{11}}{l_{p}^{24}} \frac{R_{i}}{R_{j} R_{k} R_{l}}$ \\
\hline 168 & $\frac{V_{s}^{5}}{g^{16}} \frac{s_{k}}{s_{i} s_{j}}$ & $\frac{V_{R}^{3} R_{11}}{l_{p}^{27}} \frac{R_{i} R_{j}}{R_{k}}$ \\
\hline 70 & $\frac{V_{s}^{6}}{g^{18} s_{i} s_{j} s_{k} s_{l}}$ & $\frac{V_{R}^{3} R_{11} R_{i} R_{j} R_{k} R_{l}}{l_{p}^{30}}$ \\
\hline 8 & $\frac{V_{s}^{6} s_{i}}{g^{20}}$ & $\frac{V_{R}^{4} R_{11}}{l_{p}^{33} R_{i}}$ \\
\hline
\end{tabular}

Table 12: $d=8$, momentum multiplet: $2160(\subset 3875)$

An interesting property of the spectrum in $d=7,8$ is a "mirror symmetry" corresponding to reflection around the middle level of both momentum and flux multiplets. If we denote the SYM energy of the $I$ 'th row in tables $9-12$ by $E_{I}$, and the number of rows in a table by $K$, then one finds:

$$
E_{I} E_{K+1-I}=E_{s}^{2}, \quad I=1, \ldots, K
$$

where $E_{s}$ is the $U$ duality invariant energy discussed in section 2 , (2.2); $E_{s}=V_{s} / g^{4}$ for $d=7, E_{s}=V_{s}^{3} / g^{10}$ for $d=8$. This mirror symmetry,

$$
s_{i} \rightarrow 1 / E_{s}^{2} s_{i}
$$


(with $E_{s}$ held fixed) corresponds [32] to the central element in the U duality group $E_{d(d)}(Z)$ $(d=7,8)$; A.4 $)$ is directly related to the fact that the representations of $E_{d}$ that appear are real for those values of $d$.

\begin{tabular}{|c|c|c|}
\hline$S L(9)$ & $E_{S Y M}$ & $M$ \\
\hline 9 & $\frac{g^{2} s_{i}^{2}}{N V_{s}}$ & $\frac{1}{R_{i}}$ \\
\hline 36 & $\frac{V_{s}}{N g^{2} s_{i}^{2} s_{j}^{2}}$ & $\frac{R_{i} R_{j}}{l_{p}^{3}}$ \\
\hline 126 & $\frac{V_{s} s_{i}^{2} s_{j}^{2} s_{k}^{2} s_{l}^{2}}{N g^{6}}$ & $\frac{V_{R}}{l_{p}^{6} R_{i} R_{j} R_{k} R_{l}}$ \\
\hline 252 & $\frac{V_{s}^{3}}{N g^{10}} \frac{s_{i}^{2} s_{j}^{2}}{s_{k}^{2}}$ & $\frac{V_{R}}{l_{p}^{9}} \frac{R_{k}}{R_{i} R_{j}}$ \\
\hline 9 & $\frac{V_{s}^{5}}{N g^{14} s_{i}^{4}}$ & $\frac{V_{R} R_{i}^{2}}{l_{p}^{12}}$ \\
\hline$\ldots$ & $\ldots$ & $\ldots$ \\
\hline
\end{tabular}

Table 13: $d=9$, fluxes multiplet

\begin{tabular}{|c|c|c|}
\hline$S L(9)$ & $E_{S Y M}$ & $M$ \\
\hline 9 & $\frac{1}{s_{i}}$ & $\frac{R_{11} R_{i}}{l_{p}^{3}}$ \\
\hline 126 & $\frac{s_{i} s_{j} s_{k} s_{l} s_{m}}{g^{2}}$ & $\frac{V_{R} R_{11}}{l_{p}^{6} R_{i} R_{j} R_{k} R_{l} R_{m}}$ \\
\hline 504 & $\frac{V_{s}}{g^{4}} \frac{s_{i} s_{j} s_{k}}{s_{l}}$ & $\frac{V_{R} R_{11}}{l_{p}^{9}} \frac{R_{l}}{R_{i} R_{j} R_{k}}$ \\
\hline 1260 & $\frac{V_{s}^{2}}{g^{6}} \frac{s_{i} s_{j}}{s_{k} s_{l} s_{m}}$ & $\frac{V_{R} R_{11}}{l_{p}^{12}} \frac{R_{k} R_{l} R_{m}}{R_{i} R_{j}}$ \\
\hline 72 & $\frac{V_{s}^{2}}{g^{6}} \frac{s_{i}}{s_{j}^{2}}$ & $\frac{V_{R} R_{11}}{l_{p}^{2}} \frac{R_{j}^{2}}{R_{i}}$ \\
\hline 2520 & $\frac{V_{s}^{2}}{g^{8}} \frac{s_{i}^{2} s_{j} s_{k} s_{l} s_{m}}{s_{n}}$ & $\frac{V_{R}^{2} R_{11}}{l_{p}^{15}} \frac{R_{n}}{R_{i}^{2} R_{j} R_{k} R_{l} R_{m}}$ \\
\hline 252 & $\frac{V_{s}^{2} s_{i}^{2} s_{j}^{2} s_{k}}{g^{8}}$ & $\frac{V_{R}^{2} R_{11}}{l_{p}^{15} R_{i}^{2} R_{j}^{2} R_{k}}$ \\
\hline$\ldots$ & $\ldots$ & $\ldots$ \\
\hline
\end{tabular}

Table 14: $d=9$, momentum multiplet 


\section{References}

[1] N. Seiberg, hep-th/9705117.

[2] J. Polchinski, hep-th/9611050.

[3] T. Banks, W. Fischler, S. Shenker and L. Susskind, hep-th/9610043, Phys. Rev. D55 (1997) 5112.

[4] For a review see T. Banks, hep-th/9706168.

[5] W. Taylor, hep-th/9611042, Phys. Lett. B394 (1997) 283.

[6] L. Motl, hep-th/9701025.

[7] T. Banks and N. Seiberg, hep-th/9702187.

[8] R. Dijkgraaf, E. Verlinde and H. Verlinde, hep-th/9703030.

[9] C. Hull and P. Townsend, hep-th/9410167, Nucl. Phys. B438 (1995) 109.

[10] J. Schwarz, hep-th/9510086, Phys. Lett. B367 (1996) 97.

[11] C. Montonen and D. Olive, Phys. Lett. B72 (1977) 117.

[12] L. Susskind, hep-th/9611164.

[13] O. Ganor, S. Ramgoolam and W. Taylor, hep-th/9611202, Nucl. Phys. B492 (1997) 191.

[14] W. Fischler, E. Halyo, A. Rajaraman and L. Susskind, hep-th/9703102.

[15] E. Witten, hep-th/9507121.

[16] M. Rozali, hep-th/9702136, Phys. Lett. B400 (1997) 260.

[17] M. Berkooz, M. Rozali and N. Seiberg, hep-th/9704089.

[18] R. Dijkgraaf, E. Verlinde and H. Verlinde, hep-th/9603126, Nucl. Phys. B486 (1997) 77; hep-th/9604055, Nucl. Phys. B486 (1997) 89.

[19] N. Seiberg, hep-th/9705221.

[20] D. Berenstein, R. Corrado and J. Distler, hep-th/9704087.

[21] A. Giveon, M. Porrati and E. Rabinovici, hep-th/9401139, Phys. Rept. 244 (1994) 77.

[22] O. Aharony, hep-th/9604103, Nucl. Phys. B476 (1996) 470.

[23] P. Goddard and D. Olive, Int. J. Mod. Phys. A1 (1986) 303.

[24] U. Danielsson, G. Ferretti and B. Sundborg, hep-th/9603081, Int. J. Mod. Phys. A11 (1996) 5463.

[25] D. Kabat and P. Pouliot, hep-th/9603127, Phys. Rev. Lett. 77 (1996) 1004.

[26] M. Douglas, D. Kabat, P. Pouliot and S. Shenker, hep-th/9608024, Nucl. Phys. B485 (1997) 85.

[27] F. Hacquebord and H. Verlinde, hep-th/9707179.

[28] T. Banks, N. Seiberg and S. Shenker, hep-th/9612157, Nucl. Phys. B490 (1997) 91.

[29] T. Banks and L. Susskind, hep-th/9511193, Phys. Rev. D54 (1996) 1677.

[30] E. Martinec, hep-th/9706194.

[31] D. Kutasov and E. Martinec, hep-th/9602049, Nucl. Phys. B477 (1996) 652.

[32] A. Losev, G. Moore and S. Shatashvili, to appear. 